\title{
In vivo tumor detection with combined MR-Photoacoustic-Thermoacoustic imaging
}

\author{
Lin Huang*,\|, Wei Cai ${ }^{\dagger} \|$, Yuan Zhao*, Dan Wu*, Lei Wang*, Yuqing Wang*,

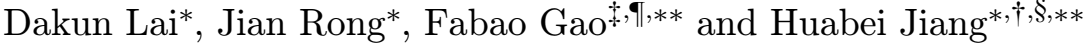 \\ *School of Physical Electronics \\ University of Electronic Science and Technology of China \\ Chengdu 610054, P. R. China \\ ${ }^{\dagger}$ Department of Biomedical Engineering \\ University of Florida, FL 32611, USA \\ ¿Department of Radiology, West China Hospital \\ Sichuan University, Chengdu, Sichuan 610041, P. R. China \\ \$hjiang@bme.ufl.edu \\ 『gaofabao@yahoo.com
}

Received 2 August 2015

Accepted 4 November 2015

Published 7 January 2016

\begin{abstract}
Here, we report a new method using combined magnetic resonance (MR)-Photoacoustic (PA)Thermoacoustic (TA) imaging techniques, and demonstrate its unique ability for in vivo cancer detection using tumor-bearing mice. Circular scanning TA and PA imaging systems were used to recover the dielectric and optical property distributions of three colon carcinoma bearing mice While a 7.0-T magnetic resonance imaging (MRI) unit with a mouse body volume coil was utilized for high resolution structural imaging of the same mice. Three plastic tubes filled with soybean sauce were used as fiducial markers for the co-registration of MR, PA and TA images. The resulting fused images provided both enhanced tumor margin and contrast relative to the surrounding normal tissues. In particular, some finger-like protrusions extending into the surrounding tissues were revealed in the MR/TA infused images. These results show that the tissue functional optical and dielectric properties provided by PA and TA images along with the anatomical structure by MRI in one picture make accurate tumor identification easier. This combined MR-PA-TA-imaging strategy has the potential to offer a clinically useful triplemodality tool for accurate cancer detection and for intraoperative surgical navigation.
\end{abstract}

Keywords: Thermoacoustic; photoacoustic; MRI; in vivo; tumor detection.

\footnotetext{
${ }^{* *}$ Corresponding authors.

${ }$ These two authors contributed equally to this study.
}

This is an Open Access article published by World Scientific Publishing Company. It is distributed under the terms of the Creative Commons Attribution 4.0 (CC-BY) License. Further distribution of this work is permitted, provided the original work is properly cited. 


\section{Huang et al.}

\section{Introduction}

Arecent cancer statistics study indicated that cancer has become a major public health problem worldwide, and a total of 1,665,540 new cancer cases and 585,720 cancer deaths are predicted to occur in the United States in 2014. ${ }^{1}$ Although cancer has a high morbidity and mortality, its early detection can effectively combat the increased incidence and death rates. In response to the requirements of early cancer detection, various functional and anatomical imaging technologies, such as magnetic resonance imaging (MRI), computed tomography (CT), positron emission tomography (PET), SPECT, and ultrasound imaging, ${ }^{2-4}$ have been routinely used for cancer detection and monitoring of therapeutic effects. However, until now, there has not existed a noninvasive and cost effective single-modality imaging method that is capable of providing all the anatomic structural and functional/physiologic information needed for early cancer diagnosis.

The power of multi-modality imaging lies within its ability to combine two or more individual modalities so that the strengths of each can be maximized in one platform. As a widely clinically used morphological imaging method, MRI offers excellent soft-tissue contrast, and uses nonionizing radiation. ${ }^{5}$ MRI reveals soft-tissue structure through the interaction of a strong magnetic field with primarily the protons present in water and fat. Although some variations of MRI such as functional MRI (fMRI) have the ability of physiological imaging, fMRI can provide only the concentration of deoxygenated hemoglobin and blood flow, and is not applicable to cancer detection due to its extremely low sensitivity. A physiologic and/or pathologic imaging modality, such as $\mathrm{PET},{ }^{6}$ can be integrated with MRI for complementary anatomical and physiological detection. A recent milestone is that MRI/PET combination has been used to image human brain. ${ }^{7}$ Such achievement suggests that multi-modality imaging of tissue structure and function will play a major role in the management of human diseases in the coming decades.

In this study, we present a novel imaging method for cancer detection, which combines MRI, photoacoustic tomography (PAT) and thermoacoustic tomography (TAT) to provide tissue anatomical structure, and optical and electric properties. While triple-modality imaging methods utilizing PAT,
MRI, and PET or Raman have been reported, ${ }^{8-11}$ to our knowledge, it is the first time that MRI, PAT, and TAT are combined for in vivo cancer detection. In addition to the morphological information obtained by MRI, PAT, and TAT offer us the possibility to access the optical and microwave absorption functional properties of tumor, which are sensitive to tumor vasculature (optical absorption coefficient) and water content (effective conductivity), respectively. It is known that tumor is always companied with larger conductivity caused by increased water/ion content and higher optical absorption due to angiogenesis (i.e., increased vasculature network). ${ }^{12,13}$ Thus, by using the quantitative PAT and TAT methods reported in Refs. 14 and 15 , we could quantify the above-mentioned functional information for accurate cancer decisionmaking. In addition, based on the photoacoustic (PA) effect first reported by Alexander Graham Bell in $1880,{ }^{16}$ TAT and PAT possess the advantages of high resolution ultrasound imaging, high microwave contrast TAT $^{17-19}$ and high optical contrast PAT. ${ }^{20-24}$ Compared to MRI-PET dual modality for in vivo tumor detection, the MRI, PAT, and TAT combined method proposed here has some advantages. This technique is more cost effective and safer for broad clinical applications. In particular, it utilizes nonionizing radiation, and does not require the use of contrast agents.

In TAT and PAT, a short nonionizing electromagnetic (EM) pulse (microwave or light wave) irradiates tissue to induce a small temperature rise ${ }^{24}$ which consequently causes thermoelastic expansion to generate thermoacoustic (TA)/PA signals. Ultrasonic transducers are employed to collect the TA/PA signals. By using an image reconstruction algorithm, TAT/PAT can then recover the EM absorption distribution inside the tissue and distinguish abnormalities from surrounding normal tissues. PAT can provide certain physiological parameters (such as the oxygen saturation of hemoglobin and the concentration of hemoglobin) with high resolution, while TAT is able to provide the distribution of absorbed microwave power loss density, which is the product of local conductivity and microwave intensity distribution within the irradiated area. As demonstrated previously, tissue conductivity directly correlates with tissue structural and functional information, such as water content and hemoglobin concentration. ${ }^{12,25}$ 


\section{Materials and Methods}

\subsection{Ethics statement}

This study was approved by the Research Ethics Board at the University of Electronic Science and Technology of China (UESTC) and the Experimental Animal Management Committee of Sichuan University. All protocols and procedures have been approved by the Animal Care Guide for the care and use of experimental animals in the UESTC, and were carried out in strict adherence to the guidelines of the Animal Care and Use Committee of Sichuan University and the Animal Ethics Committee Guidelines of the Animal Facility of the West China Hospital.

\subsection{Animal preparation}

In this study, three $(n=3)$ mice $(\mathrm{BALB} / \mathrm{C})$ were used at the age of 6-8 weeks. Heterotopic colon carcinoma was established in the groin region via subcutaneous injection with $5.0 \times 105$ C26 cells suspended in $150 \mu \mathrm{L}$ PBS. Tumor nodules typically became palpable within 4-5 days after the cell injection. Animals were treated according to the Guide for the Care and Use of Laboratory Animals of the National Institutes of Health. Tumor bearing mice were allowed 4-10 days of growth before imaging. The mice were then gas anesthetized with isofluorane ( $2 \%$ isoflurane in $100 \%$ oxygen, $1 \mathrm{~L} /$ min) using the XGI-8 Gas Anesthesia Unit (Caliper Life Sciences, USA) during MRI, and were anesthetized again using chloral hydrate $(10 \%$ concentration, $0.04 \mathrm{ml} / 10 \mathrm{~g}$ ) during PAT and TAT. Mice hair were shaved and removed with a hair removing lotion. Anesthetized mice were fixed on a homemade poly-tetrafluoroethylene holder, and three plastic tubes filled with soybean sauce attached to the holder were used as fiducial markers for the magnetic resonance (MR)-PA-TA images fusion.

\subsection{In vivo $P A / T A$ imaging systems}

The schematic of our PA/TA imaging systems is displayed in Fig. 1. A Q-switched Nd:yttriumaluminum-garnet (Nd: YAG) laser (Brilliant B, Quantel) with $1064 \mathrm{~nm}$ and $532 \mathrm{~nm}$ wavelengths, and 5-10 ns pulse duration, were used for PAT. During all the PAT experiments, a $1 \mathrm{~Hz}$ repetition frequency was applied and the radiated light intensity at the tumor surface was lower than the

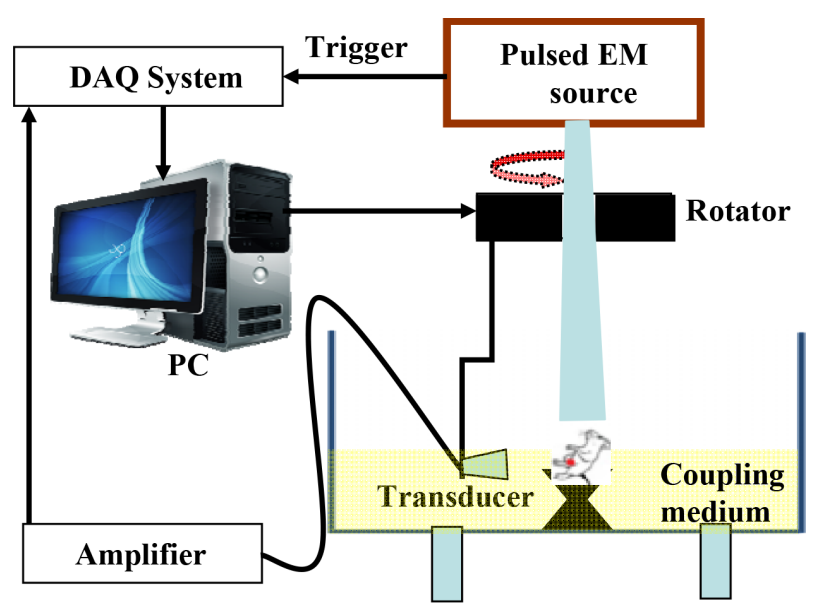

Fig. 1. Schematic of the PA/TA imaging systems. Pulsed EM source stands for laser or microwave. In PAT, water was used as a coupling medium, while transformer oil functioned as the coupling medium for TAT. DAQ: Data Acquisition.

American National Standards Institute limited safety standard of $20 \mathrm{~mW} / \mathrm{cm}^{2}$. For TAT, a $3.0 \mathrm{GHz}$ pulsed microwave from a custom-designed microwave generator (bandwidth: $50 \mathrm{MHz}$, peak power: 70-100 kW, pulse duration: $0.75 \mu \mathrm{s}$ ) was coupled into the animal. Similar to the PAT system, the TAT system works at a repetition frequency of $1 \mathrm{~Hz}$. The actual averaged microwave power density at the animal surface was about $0.32 \mathrm{~mW} / \mathrm{cm}^{2}$, which is far below the safety standard $\left(10 \mathrm{~mW} / \mathrm{cm}^{2}\right.$ at $3.0 \mathrm{GHz}$ ). For the experiments presented in this study, an unfocused immersion transducer with a central frequency of $2.25 \mathrm{MHz}$ (V323-SU, Olympus) and $5.0 \mathrm{MHz}$ (V310-SU, Olympus), was circularly scanned to receive the $\mathrm{PA}$ signals generated by mouse 1,2 , and 3 , respectively. While, only a $2.25 \mathrm{MHz}$ transducer was used for TA signals collection due to the longer microwave pulse width caused low frequency components. Thus, for our current setup, the lateral resolution of PAT and TAT can be achieved is about $0.3 \mathrm{~mm}$ and $0.5 \mathrm{~mm}$, respectively. In addition, PAT (at $532 \mathrm{~nm}$ ) and TAT, offer an imaging depth of several $\mathrm{mm}$ and $10 \mathrm{~cm}$, respectively. For effective EM wave radiation, the laser beam was expanded and homogenized when heating the sample surface by a lens and ground glass assembly. Pulsed microwaves were coupled into the sample via a standard $10 \mathrm{~dB}$ gain horn antenna $\left(114 \times 144 \mathrm{~mm}^{2}\right)$ for TA stimulation in a separated setup from PAT. The combination of TA and PA imaging was separated, due to the strong optical absorption of mineral oil used as 


\section{Huang et al.}

coupling medium in $\mathrm{TAT},{ }^{24} \mathrm{PAT}$ and TAT cannot share the same transducer and the water tank currently. However, in future clinical applications, PAT and TAT can be easily integrated for simultaneous data collection by using optical transparent oil (e.g., Lubricating oil) as coupling medium for both PAT and TAT. In that case, samples can be PA and TA imaged successively with the same transducer or arrays, so the expense and system size will be decreased. With different radiation area, during circular rotation, the transducer was placed at $8 \mathrm{~cm}$ and $11.5 \mathrm{~cm}$ apart from the scanning center for PAT and TAT, respectively. In both imaging setups, the collected signals were pre-amplified (Pre-Amp 2D, US Ultratek, Inc., USA) and converted into digital signals by a data acquisition card, which was controlled by a computer. The employed sampling rate of the data acquisition card was $50 \mathrm{MHz}$. Synchronization and control of the hardware were realized by a Labview program.

During all the experiments, mice were placed on a home-made holder and immersed into a warmed $\left(32^{\circ} \mathrm{C}\right)$ coupling medium (i.e., water for PAT, and transformer oil for TAT) for effective ultrasound signal collection after MRI scanning. A commercial rotary stage (RSA 100, Beijing Zolix Instruments CO., LTD, China) rotated the transducer relative to the center of the target. One set of data for 180 positions was acquired when the receiver was scanned circularly over $360^{\circ}$ with a step size of $2^{\circ}$. According to the $1 \mathrm{~Hz}$ repetition frequency used in both PAT and TAT, the total scanning time was $3 \mathrm{~min}$ for each imaging. Due to the strong light and microwave absorption of tumor, the systems provided a good signal to noise ratio $(\mathrm{SNR}>4)$ for both PAT and TAT, so data averaging was not necessary in this study. One shortcoming of current system is the long data acquisition time, due to the limited $1 \mathrm{~Hz}$ repetition frequency of the microwave source and laser used for data acquisition, which would hinder its way to clinical application. In our next generation PA/TA system, a considerably higher repetition frequency (e.g., $100 \mathrm{~Hz}$ ) microwave source and laser will be utilized to guarantee fast, reliable data collection, and to realize real-time imaging.

\subsection{In vivo $M R I$}

The animal experiments were performed using a 7.0-T MRI unit and a mouse body volume coil
(Bruker, Germany) at 4 and 10 days after tumor cells injection. This system can provide a spatial resolution of about $10.0 \mu \mathrm{m}$ for full-body small animal imaging. After anesthesia, the animal was laid on the scanning bed with the tumor positioned in the center of the coil. Because the colon carcinoma cells were implanted into one side of the abdomen, and the contralateral side was used as control, the position of the tumor could be defined via the difference in MRI signals from the left and right abdomen of the mice.

\subsection{Histology}

After the scanning of all three imaging modalities, the animals were sacrificed for histological evaluations. Tumors were fixed in $10 \%$ neutral buffered formalin. After that, transverse resected tumors were embedded in paraffin, sectioned, and stained with hematoxylin-eosin.

\subsection{Image processing}

PAT and TAT images were reconstructed by a Delay \& Sum program ${ }^{26}$ implemented in Matlab (Massachusetts, USA). MRI images were recovered by the manufacturer provided software. The combined MR-PA-TA images were merged by Amira (FEI Company, USA). ${ }^{27}$ Three soybean sauce filled plastic tubes (3.0 $\mathrm{mm}$ in diameter each) used as fiducial markers, which were fixed on the home-made holder with hot melt glue, were used to co-register the MR-PA-TA images. In each modality, the markers were clearly shown, and the soybean sauce surface was maintained at the same height as the tumor surface during all the experiments to ensure that MRI/PAT/TAT scanned the same slice of the tumor. For MR imaging, coronal T2-weighted fast spin echo images with fat suppression (TR/TE/slice thickness $2500 / 33 \mathrm{~ms} / 1 \mathrm{~mm}$, FOV $3.0 \times 3.0 \mathrm{~cm}^{2}$, and matrix of $256 \times 256$ resulted in an in-plane resolution of $0.137 \times 0.137 \mathrm{~mm}^{2}$ ) and $\mathrm{T} 1$-weighted spin echo images (TR/TE/slice thickness 500/ $8.3 \mathrm{~ms} / 1 \mathrm{~mm}$ ) were obtained. T2 scanned MRI slices were used for the MR-PA-TA image fusion.

Since the fiducial markers were successfully recovered in all three different images, the recovered tumor PAT and TAT images were reprocessed to have the same spatial scale as MRI. After that, the MRI, PAT and TAT image data were input into Amira work space for image fusion. According to 
the relative position of each marker, we were able to manually find the exact alignment for the MR-PATA image co-registration. An animation of the 3D MR image rendering with an overlay of the fused MR-PA-TA 2D image slices can be viewed in Media 1.

\section{Results and Discussion}

For MR-PA-TA image fusion, the tomographically recovered PAT and TAT slices corresponded to the circular scanning plane of the transducer, which is similar to the coronal plane for MRI. In vivo $\mathrm{MR}-$ PA-TA combined triple-modality images of tumor at 4 and 10 days after the implantation of cancer cells are shown in Figs. 2(a)-2(d) and 2(e)-2(h), respectively. After 4 days of tumor growth (early tumor development), the tumor reached a size of approximately $4.0 \times 1.5 \times 2.0 \mathrm{~mm}^{3}$ as determined by MRI. The coronal MRI slice shown in Fig. 2(a) clearly delineates the tumor margins with a gray signal. However, as indicated in Fig. 2(b), the tumor was not obviously detected in PAT image at this stage of tumor growth. This could be partly due to the use of $1064 \mathrm{~nm}$ wavelength for PAT as at this wavelength the optical absorption/blood content in this early tumor might not be sufficiently higher than its surrounding tissues. Similar results were also reported ${ }^{28}$ for a 3 days tumor inoculated rat with $1064 \mathrm{~nm}$ PAT system. On the other hand, since early tumor development is always accompanied by higher water content and induced larger local conductivity ${ }^{29}$ the tumor is notably detected in TAT image [Fig. 2(c)]. We also see that overlaid with TAT image, MRI contrast is enhanced with the overlaid TAT image.

The same mouse was scanned after 10 days of tumor cells injection. Compared to the images shown in Figs. 2(a)-2(c), we note that as the tumor progressively grew larger, MR imaging contrast was decreased. Due to the lack of necessary histological evaluations, we cannot explain why this is the case. This is demonstrated by the MRI slice given in Fig. 2(e). Meanwhile, we observed that the tumor grew toward the mouse to become a deeply embedded tumor 10 days after tumor cells injection, strong TAT artifacts appeared [Figs. 2(g) and 2(h)] larger due to the increased background area surrounding the tumor being TA stimulated at this time point. PAT image shown in Fig. 2(f), however, revealed clearer tumor margins and borders and high tumor-to-background contrast when overlaid with MRI. This could be due to the increased tumor vasculature at this stage of tumor growth, as evidenced by the histology analysis given in Figs. 2(i) and 2(j). Further, Fig. 2(i) also shows invasive growth of malignant tumor cells towards the surrounding normal tissue (red arrow) where it is noted that the tumor capsule integrity was destroyed. At a higher magnification [Fig. $2(\mathrm{j})]$, the frontier cells at the tumor margin infiltrated into normal tissue, where the capillaries and microvessels (yellow arrow) became particularly intensive to satisfy the needs of tumor growth.

We also examined a suspected lamp-like tumor with an MRI determined size of $7.0 \times 5.0 \mathrm{~mm}^{2}$ in lampshade, and $3.8 \times 2.0 \mathrm{~mm}^{2}$ in lamp cap. As presented in Fig. 3(a), the MR image revealed the macroscopic shape of the suspected tumor area. The division was also observed between the lamp cap and shade. However, because of the limited contrast, the MR image cannot clearly delineate the tumor margins and further predict the tumor proliferation tendency. While, as displayed in Figs. 3(b) and $3(\mathrm{~d})$, the tumor margins are partially enhanced by PA and TA signals in spots around the tumor, and the tumor boundary (see the line indicated by "1"' in Fig. 3(b), and the bottom part of the tumor [Fig. 3(d)]. The blue dotted line in Fig. 3(b) indicates the pathological observation plane outlined in Fig. 3(e). From Fig. 3(e), we found that the lamp cap was a lymph node and an intensive vascular network was apparent on one side of the tumor mass (yellow arrow), whereas on the opposite side, tumor necrosis occurred due to rapid growth and insufficient blood supplies (blue arrow). Thus, the different physiological features between " 1 " and "1" induced the apparent discrepancy PA signals at those two points, as indicated in Fig. 3(b). In other words, PAT showed absorb distribution [Fig. 3(b)] corresponded with the histology results [Fig. 3(e)]: The highlighted signal was found in the high vessel density areas and contralateral tumor necrosis was not obviously revealed in PAT. On the other hand, in Fig. 3(c), it seems that the fused MRI and TAT image shows high imaging contrast within the lamp cap. This corresponded well with the histology validation as shown in Fig. 3(e). The coregistered MR-PA-TA image shown in Fig. 3(d) suggests that it has the potential to accurately detection cancer and to guide complete resection of 


\section{Huang et al.}

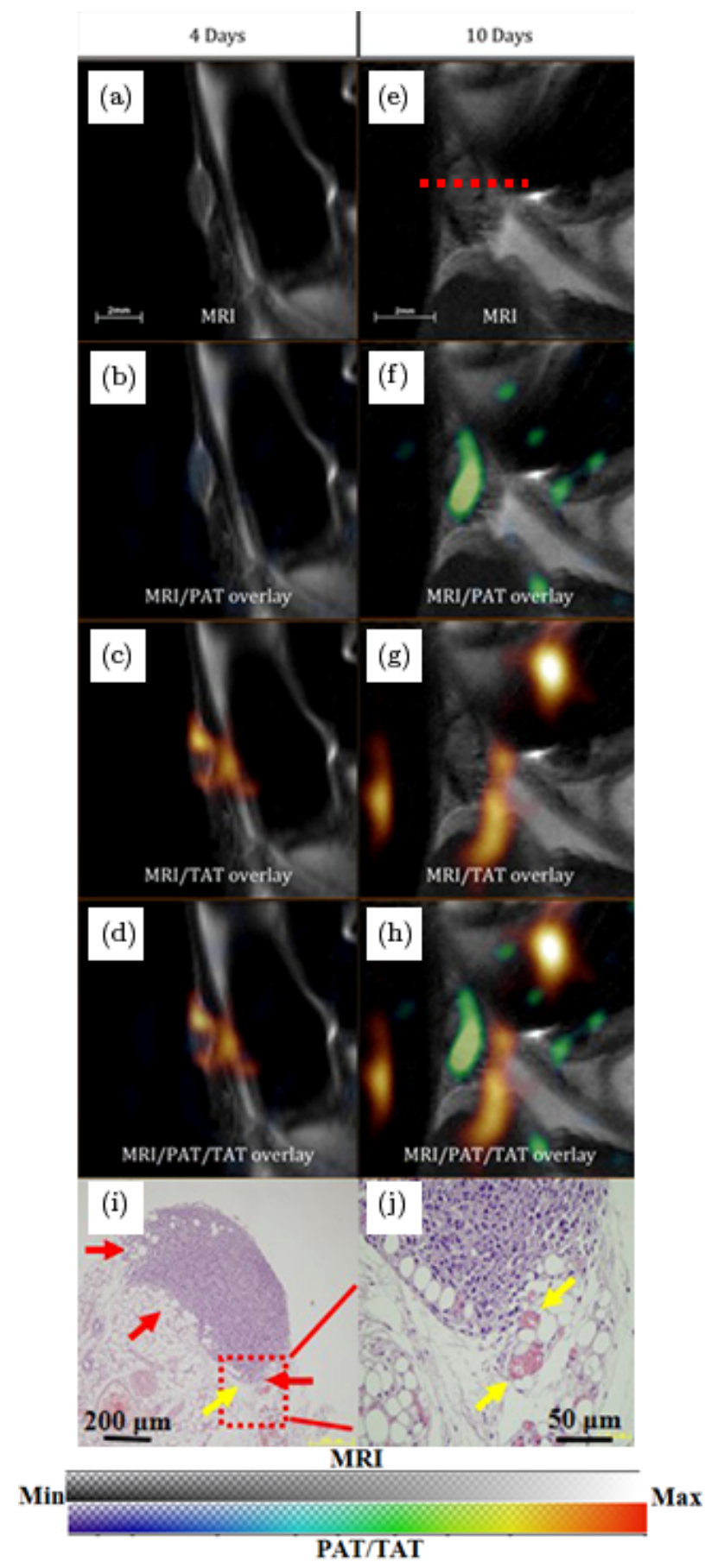

Fig. 2. Combined MR-PA-TA monitoring of early tumor development at 4 and 10 days after implantation of cancer cells. (a)-(d) and (e)-(h) represent local tumor MRI image (gray), MRI overlay with PAT image (green), MRI overlay with TAT image (orange), and triple-modality co-registered MR-PA-TA image, at 4 and 10 days following tumor cells injection, respectively. Scale bar $=2 \mathrm{~mm}$ for (a)-(h). For histology analysis, the dashed red line [I, Fig. 2(e)] indicates the histology analysis cross-section, which shows the invasive growth of malignant tumor cells towards surrounding normal tissue (red arrow) where the tumor capsule integrity was destroyed at 10 days after the cancer cells implantation. At a higher magnification in ( $\mathrm{j}$ ), the frontier cells of the tumor margin infiltrated into normal tissue, where the capillaries and microvessels (yellow arrow) became particularly intensive in order to satisfy the needs of tumor growth. The scale bar for (i) and (j) were $200 \mu \mathrm{m}$ and $50 \mu \mathrm{m}$, respectively. 


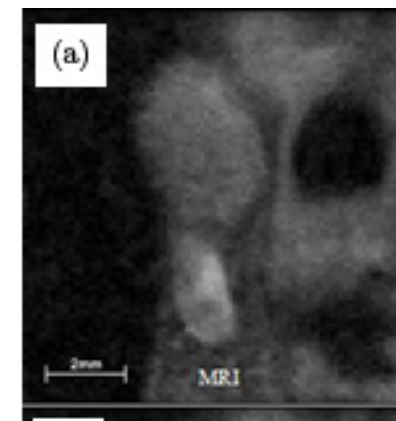

(b)
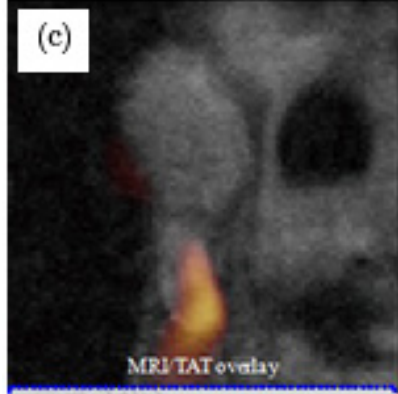

(d)

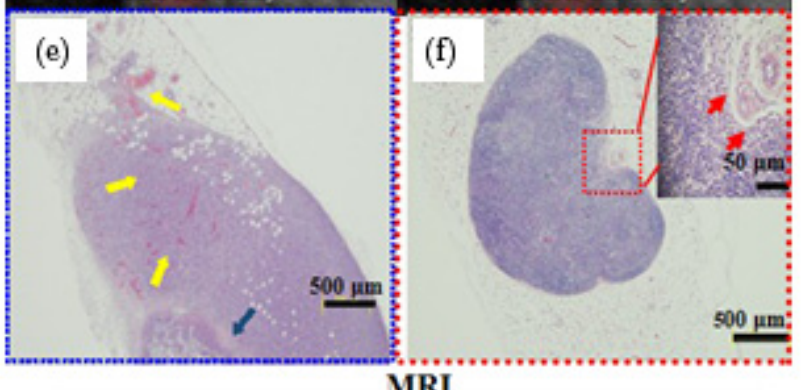

Mink

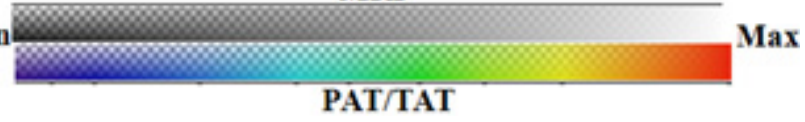

Fig. 3. Combined MR-PA-TA imaging of a lamp-like suspected tumor area. Scale bar $=2 \mathrm{~mm}$ for (a)-(d). (a) illustrates an MRI image (gray) that clearly shows suspicious lamp-like shapes, signifying the tumor area. However, the suspected area margin is not significantly different from the surrounding normal tissues without the help of PAT signal overlays (green), shown in (b). (c) displays a fused MRI and TAT image (orange), showing a high imaging contrast within the lamp camp. An enhanced TA signal expanding along within the lamp cap can also be observed. This lamp camp is otherwise invisible at lower TA signals. A co-registered MR-PA-TA image given in (d) reveals clearly delineated tumor margins and improved lamp cap contrast, and the overlaid 3D MRI, 2D PAT, and TAT slice images depicting tumor rotation (see Media 1). (e) and (f) show the dashed blue $\left(1-1^{\prime}\right)$ and red line $\left(2-2^{\prime}\right)$ indicated histology analysis cross-sections, respectively. In (e), an intensive vascular network was apparent in one side of the tumor mass (yellow arrow), whereas on the opposite side, tumor necrosis occurred (blue arrow), scale bar $=500 \mu \mathrm{m}$. The histology analysis of the dashed red line section was shown in (f) (scale bar $=500 \mu \mathrm{m})$. With the magnified red square area in the inset (scale bar $=50 \mu \mathrm{m}$ ), atypical cells emerged in sub capsule (red arrow). tumor in the absence of exogenous contrast agents with the MR-PA-TA combined triple modality method presented in this paper. For further histological validation of the tumor expanding tendency as captured by TAT, one cross-section of the lamp camp along the red dotted line in Fig. 3(d) is displayed in Fig. 3(f). A magnified red square area was placed into the inset in Fig. 3(f), where some atypical cells emerged in the sub capsule (red arrow). These cells were significantly different from the surrounding lymphocytes, featured by polymorphic nuclei and polymorphism. Thus, a lymph node metastasis was confirmed. The observed abnormal signals in both PAT and TAT also indicate that our MR-PA-TA combined triple modality method has the potential to enhance the sensitivity of MRI for lymph node detection. The 3D MRI, overlaid with $2 \mathrm{D}$ PAT, and TAT slice images depicting tumor rotation can be viewed in Media 1.

As Siphanto et al. ${ }^{28}$ reported, noninvasive and accurate imaging of angiogenesis in the tumor development has prognostic value to guide tumor anti-angiogenic therapies. It is also well known that enhanced angiogenesis imaging sensitivity could be important for tumor prognostics and the proposition of anti-angiogenic therapies. ${ }^{31,32}$ To evaluate the feasibility of our combined MR-PA-TA method for improved imaging of tumor angiogenesis without any contrast agents, we imaged the tumor surface blood vessels in a mouse. In this case, a $532 \mathrm{~nm}$ PAT system was used for blood vessels imaging. Meanwhile, as Kruger et al. ${ }^{30}$ indicated, a $5.0 \mathrm{MHz}$ transducer can meet the vasculature imaging required spatial resolution. Hence, we used a $532 \mathrm{~nm}$ PAT system with a $5 \mathrm{MHz}$ transducer for the imaging of tumor surface blood vessels [see Fig. 4(b)]. Due to the color of blood, the blood vessels imaged are visible to the naked eye, as revealed in Figs. 4(e) and 4(f). For comparison, the corresponding MR image of the tumor is given in Fig. 4(a) where we see no blood vessels signals. Similarly to Fig. 3(d), Figs. 4(c) and 4(d) show partially enhanced tumor margins with PA and TA signals in spots around the tumor. In order to more clearly observe the MRI revealed tumor and blood vessel distribution, a MRI 3D depiction of the rotation of the blood vessels is given in Media 2 (only a blood vessel below the tumor was shown in MRI animation). It can be further noticed from Fig. 4(c) 


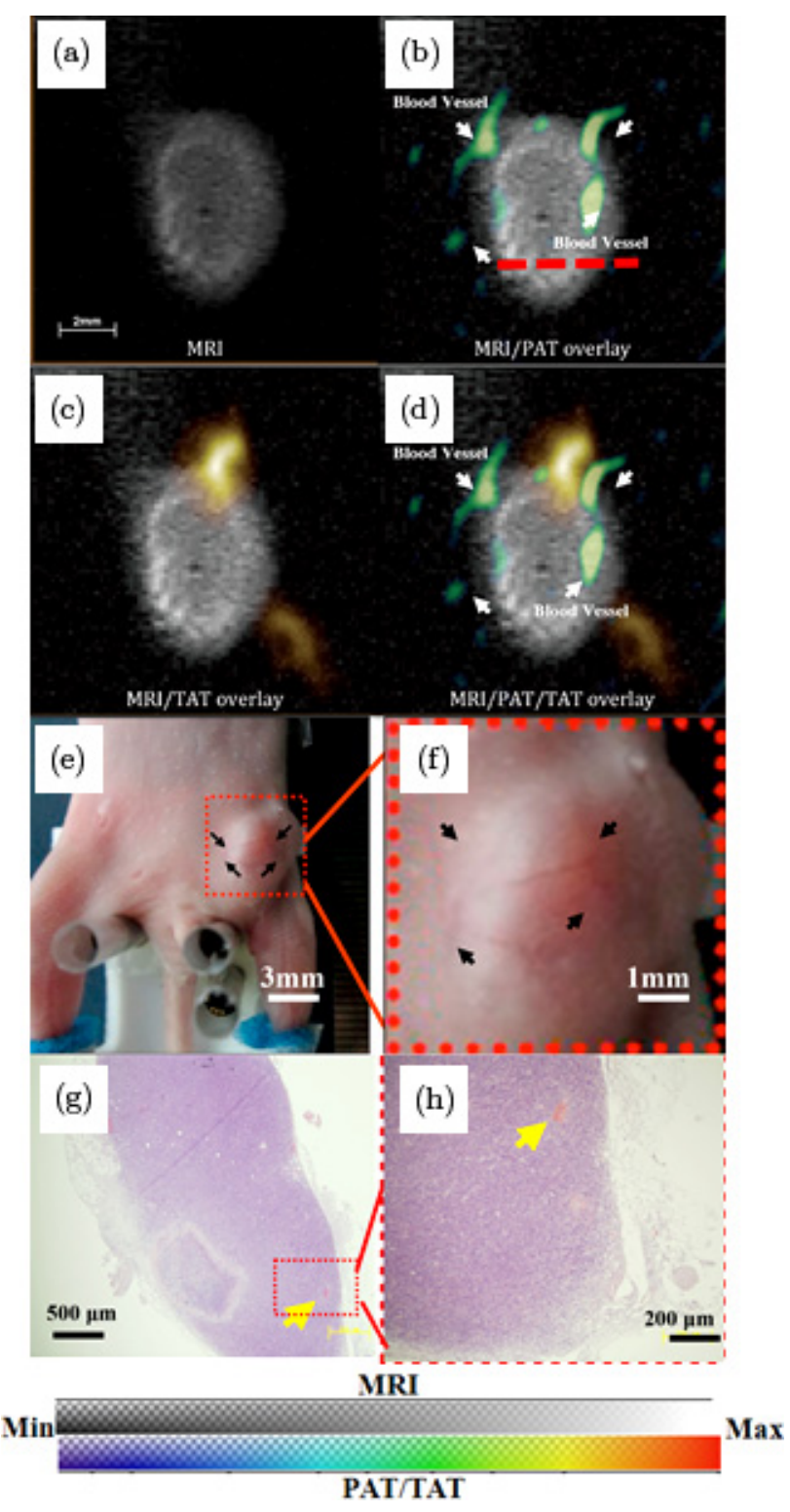

Fig. 4. Combined MR-PA-TA imaging of tumor surface vasculature. For (a)-(d), scale bar $=2 \mathrm{~mm}$. (a) Tumor MRI slice (gray) clearly displayed the tumor morphology information, while naked eye visible blood vessels indicated by the black arrow in both (e) and (f) disappeared in MRI. Overlay MRI image with $532 \mathrm{~nm}$ PAT obtained image (green) and two high PA signals were observed in (b) (as indicated by white arrow). Finger-like protrusions extending into the surrounding tissues were revealed in the MRI/TAT infused image (c), TA signal shown in orange color. (d) displayed co-registered MRPA-TA image (see Media 2). The scanned coronal slice is indicated by the red dotted block in the photograph of tumor, given in (e), and the magnified picture of the FOV (field of view) is shown in (f). Black arrow in (f) shows that the PAT recovered two high signals. Vascular histology analysis indicted in (g) present some vessels of larger size, about $100 \mu \mathrm{m}$ in diameter (yellow arrow). One of these blood vessels was magnified in (h). The scale bar for (g) and (h) was $500 \mu \mathrm{m}$ and $200 \mu \mathrm{m}$, respectively. that fused MRI/TAT image indicates high tumor contrast, compared with the surrounding normal tissues. The bright spots shown in Fig. 4(c) are the TAT captured finger-like protrusions extending into the surrounding tissues, which are evident in the histology slices [Figs. 4(g) and 4(h)]. Co-registered MRI, TAT and PAT images are shown in Fig. 4(d), and the fused image is vividly shown in a 3D MRI movie (see Media 2). The tumor microvessels shown in the histology [Fig. 4(g)] indicate their extending into the edge of the tumor, consistent with the PAT captured signals (yellow arrow). We also note the vessel's complete lumen with the magnified blood vessel histology result [Fig. 4(h)].

From some of the images presented [e.g., Fig. 2 $(\mathrm{g})$ ], we find that the overlap between the MRI revealed anatomy and the TAT and PAT indicated functional images is poor. We believe that this is mainly due to the poor longitudinal resolution of PAT and TAT. We note that the TA signal received by the transducer is the overlaid signal from the field of view within its aperture angle. Meanwhile, the tumor is irregularly shaped and surrounded by various other tissues and organs, while these other tissues and organs absorb and scatter microwave. Finally, the microwave energy is highly inhomogeneously distributed in tissue. Thus, the TA images are shown up as a group of discrete points in Figs. 2-4.

\section{Conclusions}

We have presented a novel imaging strategy using combined MR-PA-TA imaging for noninvasive, high resolution, and sensitive cancer detection. We need to point it out that, while we have shown the potential of this technique for in vivo triplemodality cancer imaging, the current study has limitations. For example, our circularly scanning data collection scheme is unsuitable for 3D TAT and PAT. New instrumentation, including economical all optical-based ultrasound acquisition systems suitable for 3D PAT and TAT hardware fusion which can finally realize true triple-modality imaging as $\mathrm{CT} / \mathrm{PET} / \mathrm{MRI}$, is currently under investigation in our lab. Results from this new instrumentation will be reported in the future. In addition, due to the high magnetic field utilized in MRI (7.0 T in this study), it is hard to integrate TAT into MRI scanner, however, it is possible to 
integrate a PAT scanner into MRI by using fiber bundles for pulsed laser propagation and PA signal detection. In this case, the TA and PA dual modality $3 \mathrm{D}$ image can be obtained first before the patient is placed into the MRI scanner, and the MRI and PA dual modality $3 \mathrm{D}$ image can then be acquired.

\section{Acknowledgment}

The authors would like to thank Max S. Jiang for his help in editing the manuscript. This research was partially supported by the Chinese Government's 1000-Talent Plan via the University of Electronic Science and Technology of China and by the J. Crayton Pruitt Family Endowment (to HJ), the National Natural Science Foundation of China (81130027 and 81520108014), the National "Twelfth Five-Year" Plan for Science \& Technology Support (2012BAI23B08) and the National Basic Research Program of China (973 Program, 2011CB935800) (to FG).

\section{Appendix}

Media 1: The overlaid 3D MRI, 2D PAT, and 2D TAT slice images depicting the rotation of the tumor: The co-registered MR-PA-TA imaging with 3D rendered MRI images showing good co-localization.

Media 2: 3D MRI depicting rotation of the blood vessels: An overlay of co-registered PAT and TAT images over the MRI angiogenesis image, indicating enhanced tumor angiogenesis sensitivity.

\section{References}

1. R. Siegel, J. M. Ma, Z. H. Zou, A. Jemal, "Cancer statistics," Ca-Cancer. J. Clin. 4(1), 9-29 (2014).

2. S. J. Gwyther, "New imaging techniques in cancer management," Ann. Oncol. 116(suppl 2), ii63-ii70 (2005).

3. M. Rudin, R. Weissleder, "Molecular imaging in drug discovery and development," Nat. Rev. Drug. Discov. 2, 123-131 (2003).

4. D. W. Townsend, "Multimodality imaging of structure and function," Phys. Med. Biol. 53(4), R1-R39 (2008).

5. H. Zaidi, A. D. Guerra, "An outlook on future design of hybrid PET/MRI systems," Med. Phys. 38 (10), 5667-5689 (2011).

6. T. E. Yankeelov, T. E. Peterson, R. G. Abramson, D. Izquierdo-Garcia, L. R. Arlinghaus, X. Li, N. C.
Atuegwu, C. Catana, H. C. Manning, Z. A. Fayad, J. C. Gore, "Simultaneous PET-MRI in oncology: A solution looking for a problem?" Magn. Reson. Imaging 30, 1342-1356 (2012).

7. M. Schmand, M. Z. Burbar, J. Corbeil, N. Zhang, C. Michael, L. Byars, L. Eriksson, R. Grazioso, M. Martin, A. Moor, J. Camp, V. Matschl, R. Ladebeck, W. Renz, H. Fischer, H. K. Jattke, G. Schnur, N. Rietsch, B. Bendriem, W. D. Heiss, "Brain PET: First human tomograph for simultaneous (functional) PET and MR imaging," J. Nucl. Med. 48, 45P (2007).

8. C. X. Qin, K. Cheng, K. Chen, X. Hu, Y. Liu, X. L. Lan, Y. X. Zhang, H. G. Liu, Y. D. Xu, L. H. Bu, X. H. Su, X. H. Zhu, S. X. Meng, Z. Cheng, "Tyrosinase as a multifunctional reporter gene for Photoacoustic/MRI/PET triple modality molecular imaging," Sci. Rep. 3, 1490 (2013).

9. H. Qin, T. Zhou, S. H. Yang, Q. Chen, D. Xing, "Gadolinium(III)-gold nanorods for MRI and photoacoustic imaging dual-modality detection of macrophages in atherosclerotic inflammation," Nanomedicine 8(10), 1611-1624 (2013).

10. M. F. Kircher, A. Zerda, J. V. Jokerst, C. L. Zavaleta, P. J. Kempen, E. Mittra, K. Pitter, R. M. Huang, C. Campos, F. Habte, R. Sinclair, C. W. Brennan, I. K. Mellinghoff, E. C. Holland, S. S. Gambhir, "A brain tumor molecular imaging strategy using a new triple-modality MRI-photoacousticRaman nanoparticle," Nat. Med. 18, 829-834 (2012).

11. S. L. Boucharda, M. S. Anwarb, G. L. Liu, B. Hannc, Z. H. Xie, J. W. Gray, X. D. Wang, A. Pines, F. Q. F. Chen, "Picomolar sensitivity MRI and photoacoustic imaging of cobalt nanoparticles," Proc. Natl. Acad. Sci. USA 106(11), 1085-4089 (2009).

12. M. Lazebnik, L. McCartney, D. Popovic, C. B. Watkins, M. J. Lindstrom, J. Harter, S. Sewall, A. Magliocco, J. H. Booske, M. Okoniewski, S. C. Hagness, "A large-scale study of the ultrawideband microwave dielectric properties of normal breast tissue obtained from reduction surgeries," Phys. Med. Biol. 52(2637), 2637-2656 (2007).

13. L. V. Wang, S. Hu, "Photoacoustic tomography: In vivo imaging from organelles to organs," Science 335(6075), 1458-1462 (2012).

14. Z. Yuan, H. B. Jiang, "Quantitative photoacoustic tomography: Recovery of optical absorption coefficient maps of heterogeneous media," Appl. Phys. Lett. 88, 231101 (2006).

15. L. Yao, G. F. Guo, H. B. Jiang, "Quantitative microwave-induced thermoacoustic tomography," Med. Phys. 37(7), 3752-3759 (2010).

16. A. G. Bell, "On the production and reproduction of sound by light," Am. J. Sci. 20, 305-324 (1880). 
17. L. Huang, J. Rong, L. Yao, W. Z. Qi, D. Wu, J. Y. $\mathrm{Xu}$, H. B. Jiang, "Quantitative thermoacoustic tomography for ex vivo imaging conductivity of breast tissue," Chinese. Phys. Lett. 30(12), 1243011-1243014 (2013).

18. L. Huang, L. Yao, L. Liu, J. Rong, H. B. Jiang, "Quantitative thermoacoustic tomography: Recovery of conductivity maps of heterogeneous media," Appl. Phys. Lett. 101, 244106 (2012).

19. Z. Ji, C. G. Lou, S. H. Yang, D. Xing, "Three-dimensional thermoacoustic imaging for early breast cancer detection," Med. Phys. 39(11), 6738-6744 (2012).

20. L. Z. Xiang, L. J. Ji, T. Zhang, B. Wang, J. J. Yang, Q. Z. Zhang, M. Jiang, J. L. Zhou, P. R. Carney, H. B. Jiang, "Noninvasive real time tomographic imaging of epileptic foci and networks," Neuroimage 66, 240-248 (2012).

21. H. Qin, T. Zhou, S. H. Yang, D. Xing, "Fluorescence quenching nanoprobes dedicated to in vivo photoacoustic imaging and high-efficient tumor therapy in deep-seated tissue," Small 11(22), 2675-2686 (2015).

22. J. Zhang, S. H. Yang, X. R. Ji, Q. Zhou, D. Xing, "Characterization of lipid-rich aortic plaques by intravascular photoacoustic tomography: Ex vivo and in vivo validation in a rabbit atherosclerosis model with histologic correlation," J. Am. Coll. Cardiol. 64(4), 385-390 (2014).

23. S. H. Yang, D. Xing, Q. Zhou, Z. L. Xiang, Y. Q. Lao, "Functional imaging of cerebrovascular activities in small animals using high-resolution photoacoustic tomography," Med. Phys. 34(8), 32943301 (2007).

24. M. Pramanik, G. Ku, C. H. Li, L. H. V. Wang, "Design and evaluation of a novel breast cancer detection system combining both thermoacoustic (TA) and photoacoustic (PA) tomography," Med. Phys. 35(6), 2218-2223 (2008).

25. X. T. Zhang, D. D. Yan, S. N. Zhu, B. He, "Noninvasive imaging of head-brain conductivity profiles using magnetic resonance electrical impedance imaging," IEEE. Eng. Med. Biol. Mag. 27(5), 78-83 (2008).

26. C. G. A. Hoelen, F. F. M de Mul, "Image reconstruction for photoacoustic scanning of tissue structures," Appl. Opt. 39(31), 5872-5883 (2000).

27. L. Z. Xiang, B. Wang, L. J. Ji, H. B. Jiang, "4-D photoacoustic tomography," Sci. Rep.3(1113) (2013).

28. R. I. Siphanto, K. K. Thumma, R. G. M. Kolkman, T. G. Van Leeuwen, F. F. M. De Mul, J. W. Van Neck, L. N. A. Van Adrichem, W. Steenbergen, "Serial noninvasive photoacoustic imaging of neovascularization in tumor angiogenesis," Opt. Express 13(1), 89-95 (2005).

29. S. P. Poplack, K. D. Paulsen, A. Hartov, P. M. Meaney, B. W. Pogue, T. D. Tosteson, M. R. Grove, S. K. Soho, W. A. Wells, "Electromagnetic breast imaging: Average tissue property values in women with negative clinical findings," Radiology 231(2), 571-580 (2004).

30. R. A. Kruger, R. B. Lam, D. R. Reinecke, S. P. Del Rio, R. P. Doyle, "Photoacoustic angiography of the breast," Med. Phys. 37(11), 6096-6100 (2010).

31. J. Hasan, R. Byers, G. C. Jayson, "Intra-tumoural microvessel density in human solid tumours," Brit. J. Cancer 86, 1566-1577 (2002).

32. M. W. Dewhirst, B. Klitzman, R. D. Braun, D. M. Brizel, Z. A. Haroon, T. W. Secomb, "Review of methods used to study oxygen transport at the microcirculatory level," Int. J. Cancer 90(5), 237-255 (2000). 